\title{
Advances in the research field of osteoporosis in cold areas
}

\author{
Ping Zhoü, Hanlu Zhang ${ }^{\#}$, Yizhen Nie, Yimeng Zhang, Yongchen Wang
}

\begin{abstract}
While osteoporosis is a chronic disease caused by multiple factors, it is also a risk factor for fractures. At present, numerous risk factors for osteoporosis and secondary fractures have been identified, including sunlight, physical fitness, gender, age, trauma, dietary habits, tobacco, alcohol, drugs, air quality, and genetics. Despite that factors such as long winters, short daylight hours, less daily physical activity, air pollution, low calcium and high salt diet, and sedentary lifestyle could negatively impact the bones of residents in the alpine regions of northern China, the direct effect of low-temperature stimulation on bone growth and development remains unclear. In this study, by reviewing current research progress related to osteoporosis and fracture risk in northern China, we proposed appropriate preventive measures for different risk factors to reduce the occurrence of osteoporosis and fracture in cold areas of northern China.
\end{abstract}

Keywords

osteoporosis; cold areas; cold stimulation; bone metabolism

Received 5 November 2021, accepted 2 December 2021
The Second Affiliated Hospital of Harbin Medical University, Harbin 150001, China

"Corresponding author Yongchen Wang, E-mail: yongchenwang@163.com

"These authors contributed equally to this work
Osteoporosis is a systemic metabolic bone disease characterized by reduced bone mass, destruction of bone tissue microstructure, and increased bone fragility. Osteoporosis causes high morbidity, disability, and mortality rates worldwide ${ }^{[1]}$. Bone metabolism is a complex process that maintains bone homeostasis through a dynamic balance between osteoblast-mediated bone formation and osteoclast-mediated bone resorption. In addition, the underlying mechanism of osteoporosis is an imbalance in bone homeostasis ${ }^{[1]}$. The development of osteoporosis is associated with a variety of factors, including genetic and environmental factors.

With the aging of the population, the incidence of osteoporosis has significantly increased. The National Health Commission reported that the incidence of osteoporosis was $19.2 \%(6.0 \%$ for men and $32.1 \%$ for women) in people over 50 years of age, while in people aged 65 years and above, the incidence rate was $32.0 \%(10.7 \%$ for men and $51.6 \%$ for women). A disparity of the incidence existed between urban and rural areas, with a higher incidence rate in rural compared to urban areas, $16.2 \%$ in urban versus $20.7 \%$ in rural areas for people aged 50 years and above and $25.6 \%$ in urban areas versus $35.3 \%$ in rural areas for people aged 65 years and above. In addition, results from a meta-analysis showed that the incidence of osteoporosis was higher in the north than in the south on a global scale $(23.17 \% \text { vs. } 20.13 \%)^{[2]}$. longjiang Province, Inner Mongolia Autonomous Region, and Xinjiang Autonomous Region are in the cold areas of China, between $45^{\circ} 7^{\prime}$ and $53^{\circ} 3^{\prime}$ north latitude, with little sunshine, long winter days, freezing and slippery roads. Fractures and complications associated with osteoporosis cause great mental stress and heavy financial burden to patients, families, and society. Therefore, we present advances in research on osteoporosis and fracture risk in cold areas, including epidemiological data, clinical reports, and basic studies collected from PubMed. Moreover, we discussed the possible mechanisms of cold stimulation on bone metabolism, providing insights into the prevention and treatment of osteoporosis in cold areas.

\section{Epidemics}

The incidence of osteoporotic fractures has a distinct geographical distribution and weather characteristics. The total number of patients suffering from fractures in snow and ice conditions is twice as high as in non-snow and ice conditions ${ }^{[3]}$. Fracture rates are significantly higher among women aged 40-69 years. Women in this age group are at higher risk for osteoporosis and falls because they stay active during inclement weather ${ }^{[4]}$. In Minnesota, USA, severe winter weather is associated with an increased risk of distal radius fractures. 
Among women aged 65 years or older, fracture risk is 1.43 times greater on snowy days, and the relative risk is 1.97 times greater in freezing rain or ice. In Oulu, Finland, the risk of radial fracture is 2.5 times higher in winter climates ${ }^{[5]}$. These statistics fully expose that low-energy fractures in cold and inclement weather in the north significantly increase the burden and workload of hospitals. Therefore, hospitals should take active and effective measures to cope with this situation.

The frequency of osteoporotic fractures of the hip varies by country, being highest in Scandinavia and lowest in Africa, which may partly reflect ethnic differences. However, the hip fracture rate in northern Europe is 11 times higher than that in the Mediterranean region ${ }^{[6]}$. The diversity of the population composition of the Asian continent also leads to a wide variation in hip fracture rates ${ }^{[7]}$.

There are multiple explanations for this geographic variation in fracture risk, which may be related to vitamin D levels, calcium consumption, diet, and genetic factors ${ }^{[8-9]}$. Having precluded the interference of these factors, Claire ${ }^{[10]}$ found a positive correlation between osteoporotic fractures and latitude and a negative correlation between hip fracture incidence and mean temperature in women and the total population. Bias correlation analysis (removing the effect of vitamin D) showed that temperature and latitude affected the incidence of the hip fracture without any relation to vitamin D. Similarly, correcting for calcium intake did not affect the relationship between temperature and latitude with regards to the incidence of the hip fracture. On the other hand, the association between latitude and hip fracture was largely eliminated when correcting for temperature.

\section{Vitamin D levels}

Vitamin D is a lipolytic hormone that plays a key role in regulating phosphorus and calcium metabolism and bone homeostasis. The body has two ways to obtain vitamin D: ultraviolet and food. Latitude, season, climate, and ozone pollution in the atmosphere can affect the solar ultraviolet light reaching the surface of the earth, thus affecting cholecalciferol production. Vitamin $\mathrm{D}$ deficiency in children can lead to rickets, while vitamin $D$ deficiency in adults can increase serum PTH, resulting in increased bone resorption. In addition, vitamin $D$ and its receptors are essential for active calcium absorption, longitudinal bone growth, and the activity of osteoblasts(OBs) and osteoclasts ${ }^{[11]}$. Vitamin $D$ levels are affected by various factors such as age, geography, race, climate, skin type, and disease conditions. In Europe, serum $25(\mathrm{OH}) \mathrm{D}$ is positively correlated with latitude, serum 25(OH)D3 levels are higher in northern European countries than in southern European countries ${ }^{[12]}$. This correlation may be due to the high rate of sun exposure, lighter skin, and the use of multivitamins by people in northern countries ${ }^{[13]}$. A high incidence of vitamin $\mathrm{D}$ deficiency has been reported among non-Western immigrants in the Netherlands, and similar data have been obtained in the Middle East, which may be related to the lifestyle habits of local women ${ }^{[14-15]}$.

A study of the distribution of basal 25(OH)D3 levels in healthy populations from the Canary Islands $\left(28^{\circ} \mathrm{N}\right)$ showed that: (1) The median 25(OH)D3 level in a healthy population of European descent was $26 \mathrm{ng} / \mathrm{ml}$; (2) The level of 25(OH)D3 was below $30 \mathrm{ng} / \mathrm{ml}$ in $65.5 \%$ of healthy individuals, below $20 \mathrm{ng} / \mathrm{ml}$ in $23.4 \%$ individuals, and below $14.3 \mathrm{ng} / \mathrm{ml}$ in $5 \%$ individuals ${ }^{[16]}$. The population with $25(\mathrm{OH}) D 3$ levels less than $30 \mathrm{ng} / \mathrm{ml}$ is $75 \%$ in the USA, $80 \%$ in France, and $83 \%$ in China ${ }^{[17-19]}$. This result can be explained by differences in race, local solar radiation levels, and detection methods.

A multicenter cross-sectional study of urban and rural areas in seven different geographic regions of China showed that $91.2 \%$ of all subjects had vitamin $D$ insufficiency, and $61.3 \%$ had vitamin $D$ deficiency. The incidence of vitamin D deficiency was significantly higher in urban than in rural areas $(64.9 \%$ vs. $57.7 \%)$ and higher in subjects enrolled in winter than in summer (84.7\% vs. $41.3 \%$ ). Vitamin D deficiency is severe in postmenopausal women in China. Winter and urban residence has a significant association with vitamin $\mathrm{D}$ deficiency ${ }^{[20]}$.

\section{Lifestyle}

\subsection{High salt intake increases calcium loss}

Adequate salt intake is essential to maintain the osmolarity of the extracellular fluid, but excess or deficiency of sodium can seriously affect the function of various organs. High salt diet (HSD) intake has negative effects on calcium homeostasis and bone mass regulation.

HSD significantly reduces calcium reserves in women of different ethnic backgrounds ${ }^{[21-22]}$. The mechanism may be due to excess sodium interfering with the ability of the renal tubular epithelium to process calcium. At the same time, excessive salt exposure causes apoptosis of intestinal epithelial cells, leading to inflammation and calcium leakage from the epithelium, which directly affects intestinal calcium absorption. The study showed that calcium transporter protein calbindin-D28k was increased in HSD rats, while mRNA levels did not change.

HSD also alters the expression of $\mathrm{NCX} 1\left(\mathrm{Na}^{+} / \mathrm{Ca}^{2+}\right.$ exchanger 1$)$ in OBs. NCX1 has been shown to be a modulator of osteoclastmediated bone resorption and has an important role in bone 
mineralization. HSD is also involved in the expression of calcium transport-related genes, but the exact cellular and molecular mechanisms need to be further investigated ${ }^{[23]}$.

However, much of the evidence is based primarily on acute salt loading studies rather than long-term diets, which is inconsistent with bone turnover studies. In the cross-sectional and cohort studies, there was a significant positive correlation between baseline (habitual diet) urinary calcium and sodium. Shortt et al. ${ }^{[24]}$ reported that sodium stimulation does not increase urinary calcium excretion in some individuals, suggesting that there may be individual differences in salt sensitivity.

Since many factors (genetic, nutritional, hormonal, and lifestyle) are involved in maintaining calcium homeostasis, it is difficult to design human studies that adequately incorporate all important factors. More studies are needed in the future to assess: (1) the effects of various nutrients, including sodium, on bone health (especially long-term sodium intake; (2) long-term evaluation of any intervention strategy that involves reducing the intake of specific nutrients (e.g., sodium); and (3) identification of subgroups, especially in the elderly (e.g., sodium-responsive subjects). Adaptation to sodium-induced hypercalciuria may be compromised in these subjects. General prudence dictates that excessively high levels of dietary salt should be eschewed by those with raised blood pressure or a limited range of genetic disorders.

\subsection{High salt intake effects affects bone histomorphology}

A recent animal study showed that excessive salt intake led to a systemic calcium imbalance and a significant increase in trabecular porosity in the tibia and femur of rats. Quantitative computed tomography (QCT) showed a significant decrease in trabecular bone mineral density with the expansion of the tibial endosteal circumference ${ }^{[25]}$.Salt loading causes transient mechanical defects and reduced resistance to bending in the long bones of the rat. Bone histomorphometric analysis showed that salt loading led to an increase in the number of osteoclasts and a decrease in the number of OBs and osteoid volume. Therefore, chronic excess salt intake may lead to impaired bone quality and integrity through a negative calcium balance ${ }^{[26]}$.

\subsection{High salt mediates osteoblast differentiation}

Wu et al. ${ }^{[27]}$ observed that superphysiological concentrations of $\mathrm{NaCl}$ affect osteoblast differentiation and their function in mice. In the human osteoblast culture system, the expression of nuclear factor-activated T cell c1 (NFATc1) gene significant increases in anti-tartrate acid phosphatase (TRAP)-positive OBs and calcitonin receptor (CTR)-positive OBs. This result suggests a direct, activated, cell-mediated effect of increased $\mathrm{NaCl}$ concentration on OBs. The mechanism is mediated by a significant increase in mRNA levels of NFATc1 and SPI1 that are the major transcription factors associated with osteoclast differentiation.

\subsection{The acid-ash hypothesis in skeleton}

The acid-ash hypothesis indicates that low potassium intake from protein and cereals induces dietary acid load and net acid excretion (NAE), increasing urinary calcium and releasing skeletal calcium, leading to osteoporosis. However, this view has inconsistent evidence on the effects of bone mineral density (BMD), fracture risk, and markers of bone formation and resorption ${ }^{[28]}$. The inconsistency arises because other components of the diet can interfere with the effects of acid/base loading on bone. High dietary protein, calcium, magnesium, and phosphorus are all necessary for bone formation.

A meta-analysis including 16 studies did not support that urinary calcium with a higher net acid excretion (NAE) was associated with systemic calcium ion loss. No high-quality parallel studies have shown that increased dietary acid load promotes bone mineral loss from the skeleton. Changes in urinary calcium do not accurately represent calcium homeostasis ${ }^{[29]}$.

The inclusion of an analysis of calcium intake may help understand the conflicting results of acidic diets in many bone studies. In the presence of an acid-producing diet and low calcium intake, bones are eroded to provide alkali to buffer excess protons. However, in the presence of high calcium intake, an acidproducing diet may have a protective effect ${ }^{[30]}$.

For people in the north, changing the structure of their diet and sticking to a healthy dietary pattern can benefit bone health. To achieve this diet, people need to eat fruits, vegetables, whole grains, poultry, fish, nuts and legumes, and low-fat dairy products and avoid eating processed foods ${ }^{[31]}$.

\subsection{Exercises}

A lot of convincing evidence indicates that physical activity has a positive impact on bone mass and bone mineral density. Moderate to vigorous exercise for more than 20 min per day increases the trabecular bone score, total hip, and femoral neck T-score. Women aged 50 or older who engage in $30 \mathrm{~min}$ of daily exercises instead of sitting can increase BMD by about $3 \mathrm{mg} / \mathrm{cm}^{2}$ and reduce the risk of spinal osteoporosis by about $12 \%{ }^{[32]}$.

Exercise varies regionally around the world. Women are more likely to lack exercise than men. People in high-income countries, such as the United States, several European countries, and 
Japan, have a higher probability of not exercising than in lowincome countries. This phenomenon may be due to more sedentary occupations and personal motorized transportation. In developing countries, especially in the north, the lack of exercise is related to the bad weather in winter and the low availability of public spaces for exercise.

In conclusion, lifestyle changes (especially a balanced nutritional intake) and regular exercise are essential to reduce the risk of osteoporosis $^{[33]}$.

\section{Cold stimulation on the skeleton}

Ambient temperature has tremendous effects on the limb growth of endotherms. The limbs of animals kept at warmer ambient temperatures are significantly and permanently longer than those kept in the same litter at cooler temperatures. This phenomenon is similar to the ecogeographic principle described by Allen's "limb size rule": the skeletal length of limbs is correlated with temperature and latitude. This phenotypic growth plasticity may have adaptive effects on thermal physiology. Temperature can also indirectly affect bone and cartilage growth by regulating circulatory levels and the delivery pathways of essential hormones and paracrine regulators ${ }^{[34]}$.

North American Inuit and Inupiat ("Eskimos") living in colder regions have lower bone mass compared to Caucasians. They are different in the pattern and extent of aging bone loss, which may be explained by the hypothesis of interpopulation variation. Accelerated bone loss in Inuit and Inupiat is combined with higher production and utilization of thyroid hormones T4 and T3. This mechanism is one of adaptation to cold through enhanced non-chilling thermogenesis, which might be attributed to their traditional "acid- ash" diet ${ }^{[35]}$.

There is evidence that cold-induced bone loss is caused by sympathetic nervous system activation and can be attenuated by uncoupling protein (UCP1) in brown adipose tissue (BAT) via non-shivering thermogenesis (NST). However, this method cannot prevent bone loss completely. Prolonged exposure to cold temperatures can damage skeletal structures, suggesting that climate may have caused phenotypic variation in humans and other archaic humans. The direct effects of cold on cortical and trabecular bone are still unknown, and it is difficult to identify the adaptation and acclimatization of populations to their environment ${ }^{[35-36]}$

In a de-ovulatory rat model of osteoporosis, cold stress promotes calcium mobilization from bone. Older female rats exposed to a warm environment at $34^{\circ} \mathrm{C}$ showed an increase in trabecular bone volume to total tibial bone volume (BV/TV) and tibial joint density without affecting tibial cortical bone, indicating a positive effect of warm environment on trabecular bone ${ }^{[37]}$. Motyl et al ${ }^{[38]}$ suggested that cold exposure negatively affects bone remodeling in mice, which is associated with increased sympathetic tone through activation of $\beta 2$-adrenergic receptors, ultimately leading to RANKL expression in OBs to stimulate bone resorption and inhibit bone formation.

Hibernating bears do not suffer from osteoporosis despite prolonged inactivity, lack of food intake and cold exposure. Japanese scholars used macrophage colony-stimulating factor (M-CSF) and receptor activator of nuclear factor kappa-B ligand (RANKL) to induce differentiation of peripheral blood mononuclear cells (PBMCs) to OBs in hibernating black bear serum. It was found that the conversion of PBMCs to OBs was inhibited ${ }^{[39]}$.

For more than a century, the effects of environmental temperature on bone have been explored from anthropological, zoological, tissue, and cellular perspectives. However, the findings are diverse, and the mechanisms are elusive and cannot be interpreted by a single factor.

\section{Cold exposure on bone metabolism markers}

A recent study discussed the effects of single and repeated wholebody cold stimulation on men with different physical abilities, including sclerostin, osteocalcin (OC), C-terminal cross-linked telopeptide of type I collagen (CTx-I), osteoprotegerin (OPG), and free soluble receptor activator of nuclear factor $\mathrm{KB}$ ligand (sRANKL). The results showed that the first exposure to extreme cold caused significant changes in serum sclerostin and SRANKL, and these mechanisms vary among people of different fitness levels. After multiple cold stimuli, the more physically fit individuals show cold adaptation ${ }^{[40]}$.

\section{Cold stimulation on adipose-muscle-skeletal energy regulatory network}

In animal studies, deficient production of BAT in mice led to bone $\operatorname{loss}^{[41]}$. In human studies, the relationship between BAT mass and BMD in healthy volunteers was determined using coldstimulated positron emission tomography (PET) and dual-energy X-ray absorptiometry (DXA). The studies revealed that high BAT mass is positively associated with BMD in healthy women ${ }^{[42]}$. The mechanism may be that after cold exposure, the sympathetic nervous system was activated, promoting UCP-1 expression and thermogenesis in BAT. In addition, brown adipocytes can form in the subcutaneous white adipose tissue depots of rodents, and cold can stimulate changes in bone density by activating the sympathetic nervous system. Therefore, BAT may have an indirect effect on bone. It has also been shown that acute cold 
exposure promotes heat production in metabolic processes, which may improve metabolic health. However, the metabolic complexity behind this process is not fully understood ${ }^{[43]}$.

The effect of cold stimulation on bone metabolism is complex. Active bone turnover depends on energy availability/demand balance and is regulated by the cross-talk of several energyrelated hormones, adipokines (leptin, adiponectin, IGF-1), myogenic factors (irisin, FGF21), neurotransmitters (i.e., insulin, leptin, adiponectin, epinephrine/norepinephrine), and osteogenic factors (osteocalcin, BMP and PTH). Muscle metabolism, adipose tissue metabolism, and bone reconstruction are closely related and can interact to maintain energy homeostasis ${ }^{[4]]}$.

Both vascular endothelial growth factor and transient receptor potential vanilloid receptors are important components of temperature sensing. A major challenge in future studies is to understand how these regulatory factors combine, how inhibitory factors control BAT thermogenesis, and how BAT responds BAT to cold.

\subsection{UCP-1}

BAT thermogenesis is mainly mediated by UCP-1. High UCP1 gene expression induced by cold temperatures is activated through the sympathetic nervous system and $\beta$-adrenergic receptors ( $\beta A R s)$. Cold exposure induces UCP1-mediated thermogenesis-dependent glucose utilization and UCP1independent active lipid metabolism in $\mathrm{BAT}^{[45]}$. Under mild cold stress, UCP-1 activity can regulate sympathetic nervous system activity through the hypothalamic neuropeptide $Y$ (NPY) pathway and is protective for bone mass when BAT-dependent thermogenesis is required ${ }^{[46]}$.

\subsection{Irisin}

Irisin is a peptide hormone derived from the hydrolysis of a fibronectin type III structural domain (FNDC5) protein containing 5. Irisin is released into the circulation after exercise or cold exposure, stimulating white adipose tissue (WAT) browning and UCP1 expression, increasing whole-body energy expenditure through enhanced UCP1-mediated thermogenesis. It plays an important role in many tissues, including muscle, fat, and bone ${ }^{[47]}$. Recent studies have shown that irisin plays an important role in osteoporosis through a complex molecule network that regulates mitochondrial energy metabolism and inhibits oxidative stress ${ }^{[48-49]}$.

\subsection{Angiopoietin-like protein ANGPTL}

Under acute and chronic cold treatment, the expression of angiopoietin-like protein ANGPTL, UCP-1, lipoprotein lipase (LPL), and adipose triglyceride lipase (ATGL) are increased in BAT and subcutaneous white adipose tissue (SAT) ${ }^{[50]}$. ANGPTL is a secreted glycoprotein family involved in many pathophysiological processes associated with osteoporosis. ANGPTL2 inhibits preadipocyte and preosteoblast differentiation, and ANGPTL2 deficiency inhibits osteoclastogenesis by regulating the NF-KB/ MAPKs/cell cycle protein pathway ${ }^{[51]}$. ANGPL4 overexpression promotes osteoclast differentiation and bone resorption ${ }^{[52]}$. ANGPTL7 is a potent inducer of osteogenic differentiation and it directly promotes osteoblast proliferation, differentiation, and mineralization by regulating the expression of $\mathrm{BMPs}^{[53]}$.

\subsection{Transient receptor potential, TRPM8}

Transient receptor potential (TRP) channels are involved in adipose tissue thermogenesis, energy metabolism, and body weight regulation. TRP channels belong to a superfamily of 28 members that are categorized into 7 subfamilies and their functionality is to perceive various stimuli, including pain, pheromones, and temperature. TRPM 8 is a $\mathrm{Ca}^{2+}$-permeable channel directly activated by low temperature as a mature cold-sensing TRP channel. TRPM8 is expressed in BAT, and activation of TRPM8 by menthol in brown adipocytes was found to upregulate UCP1 expression. TRPM8 is also expressed in human white adipocyte cell lines, where menthol activates TRPM8 and induces UCP1 expression, mitochondrial activation, and thermogenesis ${ }^{[54]}$.

In cold environments, TRPM8 deficiency is associated with differences in bone size and shape, TRPM8-KO mice had lower bone mineral density than wild-type, with smaller bone size (femur length and cross-sectional area) and such variations are more significant in males TRPM8 is required for normal thermoregulation and bone density, while in females, TRPM8 regulates bone marrow fat content. TRPM8 may help model and remodel bones throughout the life cycle ${ }^{[55]}$.

\subsection{Peroxisome-proliferator-activated receptor-1 $\alpha$}

Bone remodeling is dependent on systemic energy metabolism. In the Misty $(\mathrm{m} / \mathrm{m})$ mouse model lacking BAT, age-related trabecular bone loss was accelerated and brown fat function (including reduced temperature, reduced Pgc1a expression, and reduced sympathetic innervation) was impaired relative to wild-type $^{(++)}$. After exposure to acute hypothermic environment $\left(4^{\circ} \mathrm{C}\right.$ for $\left.6 \mathrm{~h}\right)$, inguinal white fat in Misty mice compensates for BAT dysfunction by increasing the expression of Acadl, Pgc1a, Dio2, and other thermogenic genes ${ }^{[38]}$. Deletion of peroxisome proliferator-activated receptor (PGC-1a) promotes lipogenic differentiation of mouse skeletal stem cells (SSCs) and inhibits 
osteoblast differentiation. PGC-1a deficiency in SSCs impairs bone formation and indirectly promotes bone resorption, thereby enhancing marrow adipose tissue (MAT) accumulation. It was therefore hypothesized that PGC-1 $\alpha$ attenuates bone loss and MAT accumulation in osteoporosis ${ }^{[56]}$.

\section{6 mTOR}

Rapamycin (mTOR) coordinates cellular function through targeted regulation of the serine/threonine kinase machinery, including two structurally and functionally distinct multi-protein complexes, mTOR complex 1 (mTORC1) and mTOR complex 2 (mTORC2). Under prolonged cold exposure, BAT increases to enhance thermogenesis. This process is BAT recruitment, which plays a critical role in long-term adaptation to cold environments. mTORC1 activity in BAT is highly induced after prolonged cold exposure. This effect was also observed in response to acute cold stimuli ${ }^{[57]}$. mTORC2 has biphasic regulation in brown fat metabolism in response to mild and severe cold stimulation.

Adipocytes (ADC) and OBs are both derived from mesenchymal stem cells (MSC). Their differentiation is transcriptionally regulated and mutually exclusive. Mammalian mTOR is important in determining MSC fate, and inhibition of mTOR promotes OBs differentiation and inhibits ADC differentiation. Whether the changes of mTORC1/mTORC2 induced by cold stimulation affect osteogenic-lipogenic differentiation of bone marrow mesenchymal stem cells (BMMSCs) through molecular signaling networks is still unknown. However, The conclusions in the study of the mechanisms of exercise-regulated skeletal-skeletal muscle are clear $^{[57]}$.

\subsection{Nrf2-Keap1 signal}

It has been shown that cold exposure induces myocardial oxidative stress and apoptosis by inhibiting the Nrf2-Keap1 signaling pathway ${ }^{[58]}$. Nrf2 is also expressed in OBs, regulating bone homeostasis and driving osteoblast gene expression. The Keap1/Nrf2 protein axis promotes osteoblast differentiation by regulating intracellular reactive oxygen species signaling. In both in vitro and in vivo models, Nrf2 overexpression upregulates cytoprotective enzyme expression and reduces ROS levels, the number of anti-tartrate acid phosphatase (TRAP5b)-positive polymorphonuclear cells, marker genes for osteoclast differentiation, and bone destruction. Overexpression of Keap1 or RNAi knockdown of Nrf2 produces the opposite effects. In addition, local Nrf2 overexpression in vivo attenuates lipopolysaccharide-mediated RANKL-dependent cranial bone destruction $^{[59]}$.

\section{Cold stimulation on osteoblast}

Cold stimulation promotes osteogenic differentiation of BMMSCs by activating the p38 MAPK pathway. Zhou et al. ${ }^{[60]}$ found a positive regulatory effect of the p38 MAPK signaling system on the differentiation of mouse BMMSCs into OBs. Fujiwara et al. ${ }^{[61]}$ suggested that acute cold stimulation could activate the p38 MAPK signaling pathway and that phosphorylation of p38 MAPK affects the osteogenic differentiation of BMMSCs.

Cold stimulation enhances osteogenic differentiation of BMMSCs cells and inhibits their conversion to adipocytes by promoting the expression of Runx2. These effects can be ascribed to the activation of sympathetic nervous system by cold stimulation, which then affects $\beta 2$-adrenergic receptors on the surface of OBs through the RANKL-RANK-OPG signaling pathway, and ultimately alters bone metabolism. Another explanation is that cold stimulation causes the release of adipokines and inflammatory factors in the body, resulting in changes in bone metabolism and lipid metabolism.

Nevertheless, further studies are needed to determine whether cold stimulation affects lipid metabolism first, and then leads to changes in bone metabolism, whether bone metabolism altering lipid metabolism or whether cold stimulation simultaneously acts on both metabolic pathways.

\section{Cold stimulation on BMMSC}

It was shown that the proliferation of BMMSCs cells was not significantly affected by cold stimulation for $12 \mathrm{~h}$ or $24 \mathrm{~h}$. However, the proliferation capacity of BMMSCs cells was lower significantly after cold stimulation for $48 \mathrm{~h}$ compared with the time-matched control group. BMMSCs showed spindle-like and fibroblast-like changes. One possible cause is that hypothermia disrupts the bone marrow microcirculation to damage the endothelial cells of the microvasculature, thus decreasing the synthesis of prostacyclin (PGI2) and increasing the production of vasoactive substances. This mechanism can cause platelet adhesion and aggregation, leading to impaired bone marrow microcirculation and reduced proliferation and differentiation of BMMSCs. The other cause may be that cold stimulation directly damages BMMSCs. Temperature can induce changes in proteins and disrupt cell membranes to weaken the lipid-like function of cell membranes. Previous studies showed that the cell damage is closely related to the degree and the duration of temperature reduction. However, whether the promotion of osteogenic differentiation of BMMSCs by cold stimulation is counteracted by inhibiting proliferation and differentiation of BMMSCs 
by cold stimulation needs to be further investigated ${ }^{[62]}$.

Warm exposure $\left(34^{\circ} \mathrm{C}\right)$ prevents ovariectomy-induced bone loss by increasing trabecular bone volume and joint density and thickness, thereby improving biomechanical bone strength in adult female and young male mice. Transplant phenotypes of warmadapted microbiota replicate warmth-induced skeletal effects. Both warm and warm microbiota transplants reverse ovariectomyinduced transcriptomic changes in tibiae and increase periosteal bone formation. Metagenomics/metabolomics analysis indicates that warmth enhances bacterial polyamine biosynthesis, resulting in higher total polyamine levels in the body. The supplementation of spermine and spermidine increases bone strength ${ }^{[10]}$.

In conclusion, the effects of cold on bone metabolism are multifaceted and complex, including endoplasmic reticulum (ER) stress, mitochondria-related proteins (NRFs, ROS, and Bcl-2/ Bax), and the lipoprotein lipase (LPL) signaling pathways. These effects form an extremely complex molecular signaling network. Cold stimulation can mediate changes in the gut microbiota composition, affecting bone growth and development ${ }^{[63]}$.

\section{Conclusion}

Due to the differences in human races, baseline population characteristics, lifestyles, animal species, and modes of cold stimulation (acute, chronic, short-term, long-term, degree), the results regarding the relationship between cold and osteoporosis reported in the literature are not entirely consistent. The results of animal studies may not be fully analogous to humans, who take measures to keep warm and have sufficient food security to supply energy metabolism under cold stimulation. Moreover, the imbalance of bone metabolism caused by cold stress injury under abnormal pathophysiological conditions such as inflammatory storms and immune senescence may involve multiple factors. Further comprehensive and in-depth studies are needed. This article provides a comprehensive mechanistic framework to prevent and treat osteoporosis and osteoporotic fractures in cold regions, from clinical to basic, from anthropological to animal studies, and from cellular to energy metabolic signaling networks. In conclusion, warm exposure and a rational lifestyle are reasonable as effective prevention and treatment strategies for osteoporosis.

\section{Conflicts of interests}

Yongchen Wang is an Editorial Board Member of the journal. The article was subject to the journal's standard procedures, with peer review handled independently of this member and his research groups.

\section{Acknowledgements}

This work was supported by the Natural Science Foundation of China (72074065), Cultivation Fund for Key Scientific Research Projects of Harbin Medical University, and funded by Harbin Applied Technique and Development Project (2017RAXXJ052).

\section{References}

[1] Rachner T D, Khosla S, Hofbauer L C. Osteoporosis: now and the future. Lancet, 2011; 377: 1276-1287.

[2] Chen P, Li Z, Hu Y. Prevalence of osteoporosis in China: a metaanalysis and systematic review. BMC Public Health, 2016; 16: 1039.

[3] Al-Azzani W, Mak D A M, William R, et al. Epidemic of fractures during a period of snow and ice: has anything changed 33 years on? BMJ Open, 2016; 6(9): e010582.

[4] Johnson N, Stirling E R, Dias J J. The effect of mean annual temperature on the incidence of distal radial fractures. Journal of Hand Surgery (European Volume), 2018; 43(9): 983-987.

[5] Flinkkila T, Sirnio K, Hippi M, et al. Epidemiology and seasonal variation of distal radius fractures in Oulu, Finland. Osteoporps Int, 2011; 22(8): 2307-2312.
[6] Cheng S Y, Levy A R, Lefaivre K A, et al. Geographic trends in incidence of hip fractures: a comprehensive literature review. Osteoporps Int, 2011; 22(10): 2575-2586.

[7] Eastell R, O'Neill T W, Hofbauer L C, et al. Postmenopausal osteoporosis. Nat Rev Dis Primers, 2016; 2: 16069.

[8] Wahl D A, Cooper C, Ebeling P R, et al. A global representation of vitamin D status in healthy populations. Arch Osteoporos, 2012; 7: 155172.

[9] Balk E M, Adam G P, Langberg V N, et al. Global dietary calcium intake among adults: a systematic review. Osteoporos Int, 2017; 28: 3315-3324.

[10] Chevalier C, Kieser S, Colakoglu M, et al. Warmth prevents bone loss through the gut microbiota. Cell Metabolism, 2020; 32(4): 575-590. 
[11] Lips P. Vitamin D physiology. Prog Biophys Mol Biol, 2006; 92(1): 4-8.

[12] Vieth R. Weaker bones and white skin as adaptions to improve anthropological "fitness" for northern environments. Osteoporosis, 2020; 31: 617-624.

[13] Lips P, Duong T, Oleksik A, et al. A global study of vitamin D status and parathyroid function in postmenopausal women with osteoporosis: baseline data from the multiple outcomes of raloxifene evaluation clinical trial. J Clin Endocrinol Metab, 2001; 86(3): 1212-1221.

[14] Grootjans-Geerts I. Hypovitaminosis D: a veiled diagnosis. Nederlands Tijdschrift Voor Geneeskunde, 2001; 145(51): 2057-2060.

[15] Gannage-Yared M H, Chemali R, Yaacoub N, et al. Hypovitaminosis $\mathrm{D}$ in a sunny country: relation to lifestyle and bone markers. $J$ Bone Miner Res, 2000; 15(9): 1856-1862.

[16] Garcia-Dorta A, Medina-Vega L, Villacampa-Jimenez J J, et al. Baseline levels of vitamin $D$ in a healthy population from a region with high solar irradiation. Nutrients, 2021; 13(5): 1647.

[17] Medicine I O. Dietary reference intakes for calcium and vitamin D. Washington, DC, USA: The National Academies Press, 2011.

[18] Souberbielle J C, Massart C, Brailly-Tabard B, et al. Prevalence and determinants of vitamin $D$ deficiency in healthy French adults: the VARIETE study. Endocrine, 2016; 53(2): 543-550.

[19] Jiang W, Wu D B, Xiao G B, et al. An epidemiology survey of vitamin $D$ deficiency and its influencing factors. Med Clin (Barc), 2020; 154(1): 7-12.

[20] Xie Z J, Xia W B, Zhang Z L, et al. Prevalence of vitamin D inadequacy among Chinese postmenopausal women: a nationwide, multicenter, cross-sectional study. Frontiers in Endocrinology, 2019; 9: 782.

[21] Wigertz K, Palacios C, Jackman L A, et al. Racial differences in calcium retention in response to dietary salt in adolescent girls. Am J Clin Nutr, 2005; 81(4): 845-850.

[22] Park M, Lee J S, Lee J H, et al. Prevalence and risk factors of chronic otitis media: the Korean national health and nutrition examination survey 2010-2012. PloS One, 2015; 10(5): e0125905.

[23] van der Wijst J, Tutakhel O A Z, Bos C, et al. Effects of a highsodium/low-potassium diet on renal calcium, magnesium, and phosphate handling. Am J Physiol Renal physiol, 2018; 315(1): F110-F122.

[24] Shortt C, Madden A, Flynn A, et al. Influence of dietary sodium intake on urinary calcium excretion in selected Irish individuals. Eur $\mathrm{J}$ Clin Nutr, 1988; 42(7): 595-603.

[25] Burger H, Grobbee D E, Drueke T. Osteoporosis and salt intake. Nutr Metab Cardiovasc Dis, 2000; 10(1): 46-53.

[26] Tiyasatkulkovit W, Aksornthong S, Adulyaritthikul P, et al. Excessive salt consumption causes systemic calcium mishandling and worsens microarchitecture and strength of long bones in rats. Sci Rep, 2021; 11(1): 1850.

[27] Wu L, Luthringer B J C, Feyerabend F, et al. Increased levels of sodium chloride directly increase osteoclastic differentiation and resorption in mice and men. Osteoporos Int, 2017; 28(11): 3215-3228.

[28] Hanley D A, Whiting S J. Does a high dietary acid content cause bone loss, and can bone loss be prevented with an alkaline diet? J Clin Densitom, 2013; 16(4): 420-425.

[29] Fenton T R, Lyon A W, Eliasziw M, et al. Meta-analysis of the effect of the acid-ash hypothesis of osteoporosis on calcium balance. J Bone Miner Res, 2009; 24(11): 1835-1840.
[30] Nicoll R, Howard J M. The acid-ash hypothesis revisited: a reassessment of the impact of dietary acidity on bone. J Bone Miner Metab, 2014; 32(5): 469-475.

[31] Lombardi G, Ziemann E, Banfi G. Physical activity and bone health: what is the role of immune system? A narrative review of the third way. Front Endocrinol (Lausanne), 2019; 10: 60.

[32] Sheng B, Li X, Nussler A K, et al. The relationship between healthy lifestyles and bone health: A narrative review. Medicine (Baltimore), 2021; 100(8): e24684.

[33] Weaver C M, Gordon C M, Janz K F, et al. The national osteoporosis foundation's position statement on peak bone mass development and lifestyle factors: a systematic review and implementation recommendations. Osteoporos Int, 2016; 27: 1281-1386.

[34] Serrat M A. Environmental temperature impact on bone and cartilage growth. Compr Physiol, 2014; 4(2): 621-655.

[35] Lazenby R A. Bone loss, traditional diet, and cold adaptation in Arctic populations. Am J Hum Biol, 1997; 9(3): 329-341.

[36] Fedorenko A, Lishko P V, Kirichok Y. Mechanism of fatty-aciddependent UCP1 uncoupling in brown fat mitochondria. Cell, 2012; 151(2): 400-413.

[37] Iwaniec U T, Philbrick K A, Wong C P, et al. Room temperature housing results in premature cancellous bone loss in growing female mice: implications for the mouse as a preclinical model for age-related bone loss. Osteoporos Int, 2016; 27(10): 3091-3101.

[38] Motyl K J, Bishop K A, DeMambro V E, et al. Altered thermogenesis and impaired bone remodeling in misty mice. J Bone Miner Res, 2013; 28(9): 1885-1897.

[39] Nasoori A, Okamatsu-Ogura Y, Shimozuru M, et al. Hibernating bear serum hinders osteoclastogenesis in-vitro. PLoS ONE, 2020; 15(8): e0238132.

[40] Straburzynska-Lupa A, Cison T, Gomarasca M, et al. Sclerostin and bone remodeling biomarkers responses to whole-body cryotherapy (-110 degrees $\mathrm{C}$ ) in healthy young men with different physical fitness levels. Sci Rep, 2021; 11: 16156.

[41] Shi X M, Blair H C, Yang X, et al. Tandem repeat of C/EBP binding sites mediates PPARgamma2 gene transcription in glucocorticoidinduced adipocyte differentiation. J Cell Biochem, 2000; 76(3): 518-527.

[42] Veldhuis-Vlug A G, Rosen C J. Clinical implications of bone marrow adiposity. J Intern Med, 2018; 283(2): 121-139.

[43] Kovanicova Z, Karhanek M, Kurdiova T, et al. Metabolomic analysis reveals changes in plasma metabolites in response to acute cold stress and their relationships to metabolic health in cold-acclimatized humans. Metabolites, 2021; 11(9): 619.

[44] Lee P, Linderman J D, Smith S, et al. Irisin and FGF21 are coldinduced endocrine activators of brown fat function in humans. Cell Metab, 2014; 19(2): 302-309.

[45] Razzoli M, Emmett M J, Lazar M A, et al. Beta-Adrenergic receptors control brown adipose UCP-1 tone and cold response without affecting its circadian rhythmicity. Faseb J, 2018; 32(10): 5640-5646.

[46] Nguyen A D, Lee N J, Wee N K Y, et al. Uncoupling protein-1 is protective of bone mass under mild cold stress conditions. Bone, 2018; 106: 167-178.

[47] Calton E K, Soares M J, James A P, et al. The potential role of irisin in the thermoregulatory responses to mild cold exposure in adults. Am J Hum Biol, 2016; 28(5): 699-704.

[48] Wang F S, Wu R W, Chen Y S, et al. Biophysical modulation of 
the mitochondrial metabolism and redox in bone homeostasis and osteoporosis: how biophysics converts into bioenergetics. Antioxidants, 2021; 10(9): 1394.

[49] Kornel A, Den Hartogh D J, Klentrou P, et al. Role of the myokine irisin on bone homeostasis: review of the current evidence. International Journal of Molecular Sciences, 2021; 22(17): 9136.

[50] Arefanian H, Al-Khairi I, Khalaf N A, et al. Increased expression level of ANGPTL8 in white adipose tissue under acute and chronic cold treatment, 2021; 20: 117.

[51] Li W M, Han C L, Liu C, et al. ANGPTL2 deletion inhibits osteoclast generation by modulating NF-kappaB/MAPKs/Cyclin pathways. Biochem Biophys Res Commun, 2018; 503(3): 1471-1477.

[52] Kadomatsu T, Oike Y. Roles of angiopoietin-like proteins in regulation of stem cell activity. J Biochem, 2019; 165(4): 309-315.

[53] Lu X, Lu J, Zhang L, et al. Effect of ANGPTL7 on proliferation and differentiation of MC3T3-E1 cells. Med Sci Monit, 2019; 25: 9524-9530.

[54] Clapham D E. TRP channels as cellular sensors. Nature, 2003; 426: 517-524.

[55] Carvalho A L, Treyball A, Brooks D J, et al. TRPM8 modulates temperature regulation in a sex-dependent manner without affecting cold-induced bone loss. PLoS One, 2021; 16: e0231060.

[56] Yu B, Huo L, Liu Y, et al. PGC-1alpha controls skeletal stem cell fate and bone-fat balance in osteoporosis and skeletal aging by inducing
TAZ. Cell Stem Cell, 2018; 23(4): 193-209.

[57] Labbé S M, Mouchiroud M, Caron A, et al. mTORC1 is required for brown adipose tissue recruitment and metabolic adaptation to cold. Sci Rep, 2016; 6: 37223.

[58] Cong P, Liu Y, Liu N, et al. Cold exposure induced oxidative stress and apoptosis in the myocardium by inhibiting the Nrf2-Keap1 signaling pathway. BMC Cardiovascular Disorders, 2018; 18(1): 36

[59] Sanchez-de-Diego C, Pedrazza L, Pimenta-Lopes C, et al. NRF2 function in osteocytes is required for bone homeostasis and drives osteocytic gene expression. Redox Biology, 2021; 40: 101845.

[60] Zhou H, Yang X, Wang N, et al. Tigogenin inhibits adipocytic differentiation and induces osteoblastic differentiation in mouse bone marrow stromal cells. Mol Cell Endocrinol, 2007; 270(1-2): 17-22.

[61] Fujiwara Y, Denlinger D L. p38 MAPK is a likely component of the signal transduction pathway triggering rapid cold hardening in the flesh fly Sarcophaga crassipalpis. J Exp Biol, 2007; 210(pt 18): 3295-3300. [62] Nie Y, Yan Z, Yan W, et al. Cold exposure stimulates lipid metabolism, induces inflammatory response in the adipose tissue of mice and promotes the osteogenic differentiation of BMMSCs via the p38 MAPK pathway in vitro. Int J Clin Exp Pathol, 2015; 8(9): 10875-10886. [63] Moreno-Navarrete J M, Fernandez-Real J M. The gut microbiota modulates both browning of white adipose tissue and the activity of brown adipose tissue. Rev Endocr Metab Disord, 2019; 20(4): 387-397. 